\title{
CYP2C19 polymorphisms and clopidogrel efficacy in the secondary prevention of ischemic stroke: a retrospective observational study
}

\author{
Juanna Lin ${ }^{1}$, You Mo ${ }^{2}$, De Cai ${ }^{1}$, Dongyang Mao ${ }^{1}$, Hongbo Fu ${ }^{3}$, Duncan Wei ${ }^{1}$ \\ ${ }^{1}$ Department of Pharmacy, First Affiliated Hospital of Shantou University Medical College, Shantou, China; ${ }^{2}$ Department of Angiocarpy, First \\ Affiliated Hospital of Shantou University Medical College, Shantou, China; ${ }^{3}$ Department of Pharmacy, Second Affiliated Hospital of Shantou \\ University Medical College, Shantou, China \\ Contributions: (I) Conception and design: D Wei, H Fu; (II) Administrative support: D Mao, H Fu; (III) Provision of study materials or patients: Y \\ Mo, D Cai; (IV) Collection and assembly of data: J Lin; (V) Data analysis and interpretation: J Lin, D Wei; (VI) Manuscript writing: All authors; (VII) \\ Final approval of manuscript: All authors. \\ Correspondence to: Duncan Wei. Department of Pharmacy, First Affiliated Hospital of Shantou University Medical College, Shantou, China. \\ Email: weiduncan2012@126.com; Hongbo Fu. Department of Pharmacy, Second Affiliated Hospital of Shantou University Medical College, \\ Shantou, China. Email: sdfefhb@126.com.
}

Background: The relationship between cytochrome P450 2C19 (CYP2C19) polymorphisms and clopidogrel efficacy in patients with percutaneous coronary intervention (PCI) has been widely studied. However, the relationship between CYP2C19 polymorphisms and the response to clopidogrel in patients treated for ischemic stroke (IS) remains controversial. What's more, few data address the relevance of CYP2C19 polymorphisms in patients taking clopidogrel for secondary prevention of ischemic stroke. This study investigates whether carrying CYP2C19 loss-of-function (LOF) alleles affects the risk of recurrent stroke in IS patients.

Methods: One hundred twenty-two IS patients were CYP2C19 genotype screened and enrolled in the study from January 2016 to December 2017. Those with stroke recurrence, stroke sequelae, or bleeding diseases were excluded. The remaining 89 patients were divided into the following 2 groups: non-carriers of CYP2C19 LOF alleles ( $n=38)$ and carriers $(n=51)$ of CYP2C19 LOF alleles. The variables that could influence the rate of recurrent stroke were assessed in a multivariate analysis to determine the independent risk factors.

Results: The CYP2C19*2 and *3 alleles frequencies among the 122 patients were $31.97 \%$ and $4.10 \%$, respectively. Carriers of LOF alleles had a more significant history of hypertension compared with noncarriers $[\mathrm{n}=43 / 51(86.7 \%)$ versus $\mathrm{n}=23 / 38$ (60.5\%), $\mathrm{P}=0.01]$. In addition, the inclusion rate of aspirin in discharge medication was significantly higher for carriers than for non-carriers $[\mathrm{n}=19 / 51(37.3 \%)$ versus $\mathrm{n}=5 / 38(13.2 \%), \mathrm{P}=0.01]$. CYP2C19 LOF alleles were significantly associated with an increased risk of recurrent stroke [odds ratio (OR): 7.586; 95\% confidence interval (CI): 1.346-42.770, $\mathrm{P}=0.022$ ].

Conclusions: CYP2C19 LOF alleles may increase the risk of recurrent IS. The polymorphisms of CYP2C19 may be predictors of a poor functional outcome in patients with recurrent stroke. Instead of clopidogrel, aspirin can be prescribed as a secondary preventative measure against stroke in carriers of CYP2C19 LOF alleles.

Keywords: CYP2C19 polymorphisms; CYP2C19 LOF allele; clopidogrel efficacy; ischemic stroke

Submitted Sep 21, 2021. Accepted for publication Dec 01, 2021.

doi: 10.21037/apm-21-2905

View this article at: https://dx.doi.org/10.21037/apm-21-2905 


\section{Introduction}

Stroke is a worldwide leading cause of death and neurological disability, and puts enormous physical, psychological, and financial burdens on patients in developing and developed countries alike (1). Between 1993 and 2013, Chinese stroke morbidity showed a monotonic increase from $0.4 \%$ to $1.23 \%$. Approximately 2,500,000 people suffer their first stroke each year in China, with the recurrence rate within 1 year reaching $17.7 \%$. These recurrences are associated with higher mortality rates, greater levels of disability, and increased costs compared with first stroke events (2-4). Although reperfusion therapies are available for patients with acute ischemic stroke (IS), effective prevention (especially secondary prevention) is considered the most effective pathway for reducing the recurrence of stroke and associated deaths $(5,6)$.

Antiplatelet medications are an important secondary measure against recurring IS, reducing stroke-related mortality by preventing thrombotic IS when taken after acute IS $(2,7,8)$. Of the many antiplatelet agents, clopidogrel is commonly used alone or in conjunction with aspirin for stroke prevention in at-risk individuals and recommended as an antiplatelet drug for secondary prevention of IS. Clopidogrel is a thienopyridine prodrug, which inhibites platelet aggregation, commonly regulates the activated platelet for secondary prevention of IS and prevent early recurrence. Clopidogrel requires conversion to its active metabolite through two sequential oxdative steps, the first step leads to formation of 2-oxo-clopidogrel, followed by the conversion of 2-oxo-clopidogrel to the active metabolite. This 2-stage mediated largely by the metabolizing enzyme cytochrome P450 2C19 (CYP2C19) $(9,10)$.

CYP2C19 is one of the most important polymorphic CYP enzymes in populations worldwide. Over 25 genetic variants have been identified for the CYP2C19 gene, of which CYP2C19*2 (G681A) and CYP2C19*3 (G636A) mutations are the two most functionally important variants. They can reduce the active metabolite levels of clopidogrel, diminished platelet inhibition and contribute to a decrease in its effective function; Furthermore, patients carrying at least one CYP2C19 loss-of-function (LOF) alleles (*2, *3) had a higher rate of clinical outcomes(such as myocardial infarction or stroke, and stent thrombosis) than patients who did not have these alleles (11-13). In 2010, the United States Food and Drug Administration (US FDA) issued a box warning on clopidogrel, suggesting that the drug has a diminished effect on poor metabolizers (PM) because they carry two CYP2C19 LOF alleles (14).

If CYP2C19 genetic testing is widely adopted, it must be shown to be cost effective. Besides the cost of test itself, the newer alternative medications for carries of CYP2C19 LOF alleles (e.g., Prasugrel, ticagrelor) are more expensive than clopidogrel, and potential cost-savings from decreased cardiovascular events. Indeed, CYP2C19 genetic testing will be important to perform real-world cost and effectiveness analyses. Furthermore, CYP2C19 genotype information can be immediately available to guide anti-platelet therapy at the time it is indicated (8).

The most definitive studies showing a relationship between CYP2C19 polymorphisms and clopidogrel efficacy have been conducted in patients with acute coronary syndrome (ACS) and patients undergoing percutaneous coronary intervention (PCI) (15). However, the relationship between CYP2C19 polymorphisms and the response to clopidogrel in patients treated for IS remains controversial. Some studied have shown CYP2C19 polymorphisms may influence clopidogrel efficacy in stroke patients, and a few studies showed that CYP2C12 LOF allele carriers had poorer functional outcomes and higher risk of ischemic events versus noncarriers with ischemic stroke $(9,11)$. In this study, we selected and enrolled stroke patients who had undergone CYP2C19 genotype screening to determine the relationship between CYP2C19 polymorphisms and clopidogrel efficacy for IS. The findings of this study may guide the development of personalized clinical antiplatelet therapy for IS. We present the following article in accordance with the STROBE reporting checklist (available at https://dx.doi.org/10.21037/apm-21-2905).

\section{Methods}

\section{Ethical considerations}

The study was conducted in accordance with the Declaration of Helsinki (as revised in 2013) and was consistent with relevant guidelines and regulations for clinical studies. The study was approved by ethics board of the First Affiliated Hospital of Shantou University Medical College, China (No. 2019043). Informed consent was obtained from each patient before enrollment.

\section{Study population}

In total, 122 stroke patients underwent CYP2C19 genotype 
screening and were enrolled in the study from January 2016 to December 2017. The inclusion criteria were as follows: patient with a clinical diagnosis of IS according to the revised guideline of the 4th Cerebrovascular Disease Forum of China, confirmed by computed tomography (CT) or magnetic resonance imaging (MRI) as a minimum; patient aged 18 years or older; patient treated with clopidogrel for 5 days or longer. The exclusion criteria included: patient with recurrence or sequelae of stroke; clopidogrel contraindicated in the patient; patient platelet count greater than $450 \times 10^{9} / \mathrm{L}$ or less than $150 \times 10^{9} / \mathrm{L}$; patient taking other anticoagulation drugs, such as warfarin; patient with a recent history of active bleeding; patient diagnosed with severe kidney or liver diseases; or patient with major surgery within 1 month of the study.

\section{CYP2C19 genotyping}

CYP2C19 genotyping detection was collected from each patient's electronic medical records. Based on their CYP2C19 genotype, phenotypes were then classified as extensive metabolizers $\left(\mathrm{EM},{ }^{*} 1 /{ }^{*} 1\right)$, intermediate metabolizers (IM, ${ }^{*} 1 /{ }^{*} 2$ or $\left.{ }^{*} 1 /{ }^{*} 3\right)$, and poor metabolizers $\left(\mathrm{PM},{ }^{*} 2{ }^{*} 2,{ }^{*} 3 / * 3\right.$, or $\left.{ }^{*} 2 / * 3\right)(15)$. The CYP2C19 allelic frequency $\left({ }^{*} 1, * 2, * 3\right)$ used by Hardy-Winberg equilibrium law. Of the initial 122, we identified 89 suitable patients and classified them into the following 2 groups: non-carriers of CYP2C19 LOF alleles $\left({ }^{*} 1 /{ }^{*} 1\right)(\mathrm{n}=38)$ and carriers of CYP2C19 LOF alleles $\left({ }^{*} 1 /{ }^{*} 2,{ }^{*} 1 /{ }^{*} 3,{ }^{*} 2 /{ }^{*} 2,{ }^{*} 2 /{ }^{*} 3\right)(\mathrm{n}=51)$.

\section{Data collection and follow-up}

Clinical data, including demographic characteristics, clinical information, laboratory parameters, and medications, were collected from each patient's electronic medical records at the First Affiliated Hospital of Shantou University Medical College.

Clinical follow-up involved either a clinic visit or hospital readmission at 3, 6, 9, and 12 months after hospital discharge. The primary clinical findings in follow-up were arteriosclerotic encephalopathy, recurrent stroke, and bleeding. All patients with symptoms during the visits and interviews were evaluated, and resident physicians took records.

\section{Statistical analysis}

All statistical analyses were performed using SPSS 16.0
(SPSS Inc., Chicago, IL, USA), and the Hardy-Weinberg equilibrium for genotype frequencies was tested using the chi-square test. Measurement data were represented as the mean \pm standard deviation (SD), and groups were compared using an independent-samples t-test. The categorical data were presented as percentages and assessed using the chisquare test. Variables found to have a significant association with the risk of recurrent IS were then analyzed using multivariate logistic regression analysis. In the logistic regression model, the CYP2C19 genetic variants were coded as: (I) 0 for absence of CYP2C19 LOF alleles $\left({ }^{*} 1 /{ }^{*} 1\right)$; or (II) 1 for carriers of 1 or 2 LOF alleles $\left({ }^{*} 1 /{ }^{*} 2,{ }^{*} 1 /{ }^{*} 3\right.$, $\left.* 2 /{ }^{*} 2,{ }^{*} 2 / 3\right)$. A P-value $<0.05$ was considered statistically significant.

\section{Results}

\section{representative MR imaging of IS patients}

It was showed that ischemic stroke was diagnosed by computed tomography (CT) or magnetic resonance imaging (MRI). The results showed the study have used mainly MRI to diagnose IS, and provided representative images of MRI of IS (Figure 1).

\section{Genotypic and allelic frequencies and phenotypes of IS patients}

The initial group of 122 IS patients underwent CYP2C19 genotype screening from July 2016 to December 2017 at our hospital. We found that $54.92 \%$ of the patients carried at least 1 CYP2C19 LOF allele, with $17.21 \%$ carrying 2 LOF alleles. The CYP2C19*1/*1 genotype was the most predominant among the patients $(45.08 \%)$, followed by the CYP2C19*1/*2 genotype (32.79\%). We also calculated genotype frequencies as follows: the CYP2C19*1 allelic frequency was $63.93 \%$ (including ${ }^{*} 1 /{ }^{*} 1,{ }^{*} 1 /{ }^{*} 2,{ }^{*} 1 /{ }^{*} 3$ ); the CYP2C19*2 allelic frequency was $31.97 \%$ (including ${ }^{*} 1{ }^{*} 2$, $* 2 / * 2, * 2 / * 3)$; and the CYP2C19*3 allelic frequency was $4.10 \%$ (Table 1).

\section{Clinical characteristics and baseline laboratory parameters}

Of the 122 patients first selected, 33 were excluded in accordance with the exclusion criteria (20 for recurrence, 8 for sequelae, and 5 for other reasons). According to differences in CYP2C19 genotypes and phenotypes, the remaining 89 patients were divided into the following 2 

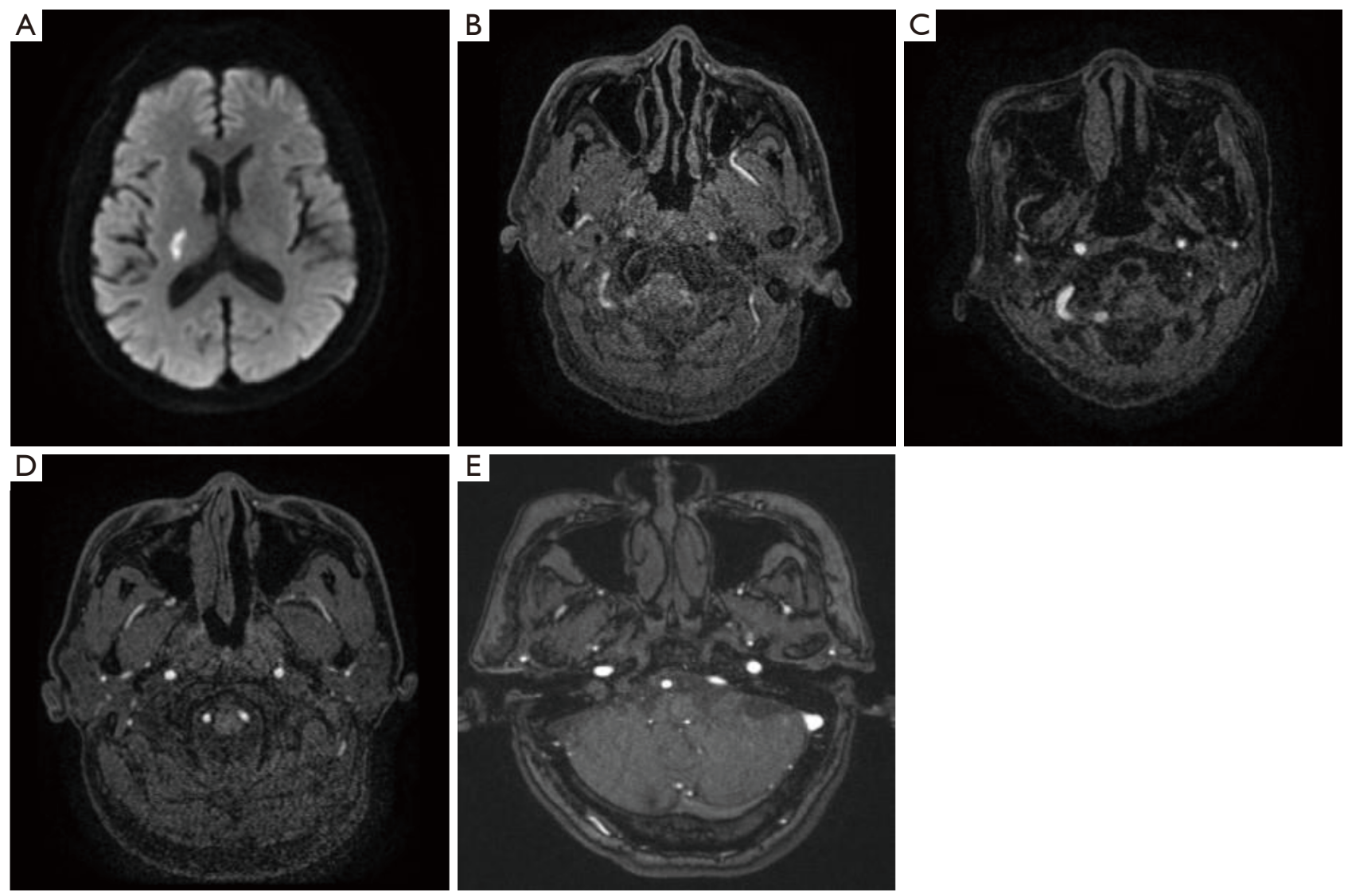

Figure 1 Representative MR imaging of IS patients. (A) Patient with CYP2C19*1/*1, the left lateral ventricle had sporadic dots with slightly longer T1 signal, longer T2 signal, DWI higher signal. (B) Patient with CYP2C19*1/*2, the right basal ganglia showed sporadic patches with slightly longer T1 signal, longer T2 signal, T2-FLAIR higher signal; the left maxillary sinus had sporadic dots. (C) Patient with CYP2C19*1/*3, The bilateral frontal lobe, ventricle, left cerebral ganglion and left basal ganglia showed sporadic dots and patches with slightly longer T1 signal, longer T2 signal, FLAIR higher signal. (D) Patient with CYP2C19*2/*2, the right pons showed sporadic patches with slightly longer T1 signal, longer T2 signal, FLAIR higher signal. (E) Patient with CYP2C19*2/33, The bilateral ventricles, basal ganglia, thalamus and pons showed Multiple patches with slightly longer T1 signal, longer T2 signal, FLAIR higher signal. MR, magnetic resonance; IS, ischemic stroke; DWI, diffusion-weighted imaging; FLAIR, fluid-attenuated inversion recovery.

Table 1 Genotypic and allelic frequencies and phenotypes of IS patients

\begin{tabular}{|c|c|c|c|c|c|c|}
\hline Gene & Genotype & Distribution, n (\%) & Phenotype & Allele & Frequency, \% & $P$ value \\
\hline \multirow{4}{*}{ CYP2C19 } & ${ }^{*} 1 /{ }^{*} 2$ & $40(32.79)$ & $\mathrm{IM}$ & *2 & 31.97 & \\
\hline & $* 1 / * 3$ & $6(4.92)$ & $\mathrm{IM}$ & *3 & 4.10 & \\
\hline & $* 2 /{ }^{*} 2$ & 17 (13.93) & PM & & & \\
\hline & ${ }^{*} 3 /{ }^{*} 3$ & $0(0)$ & PM & & & \\
\hline
\end{tabular}

EM, extensive metabolizers; IM, intermediate metabolizers; PM, poor metabolizers. 
Table 2 Baseline demographic and clinical characteristics of the study population

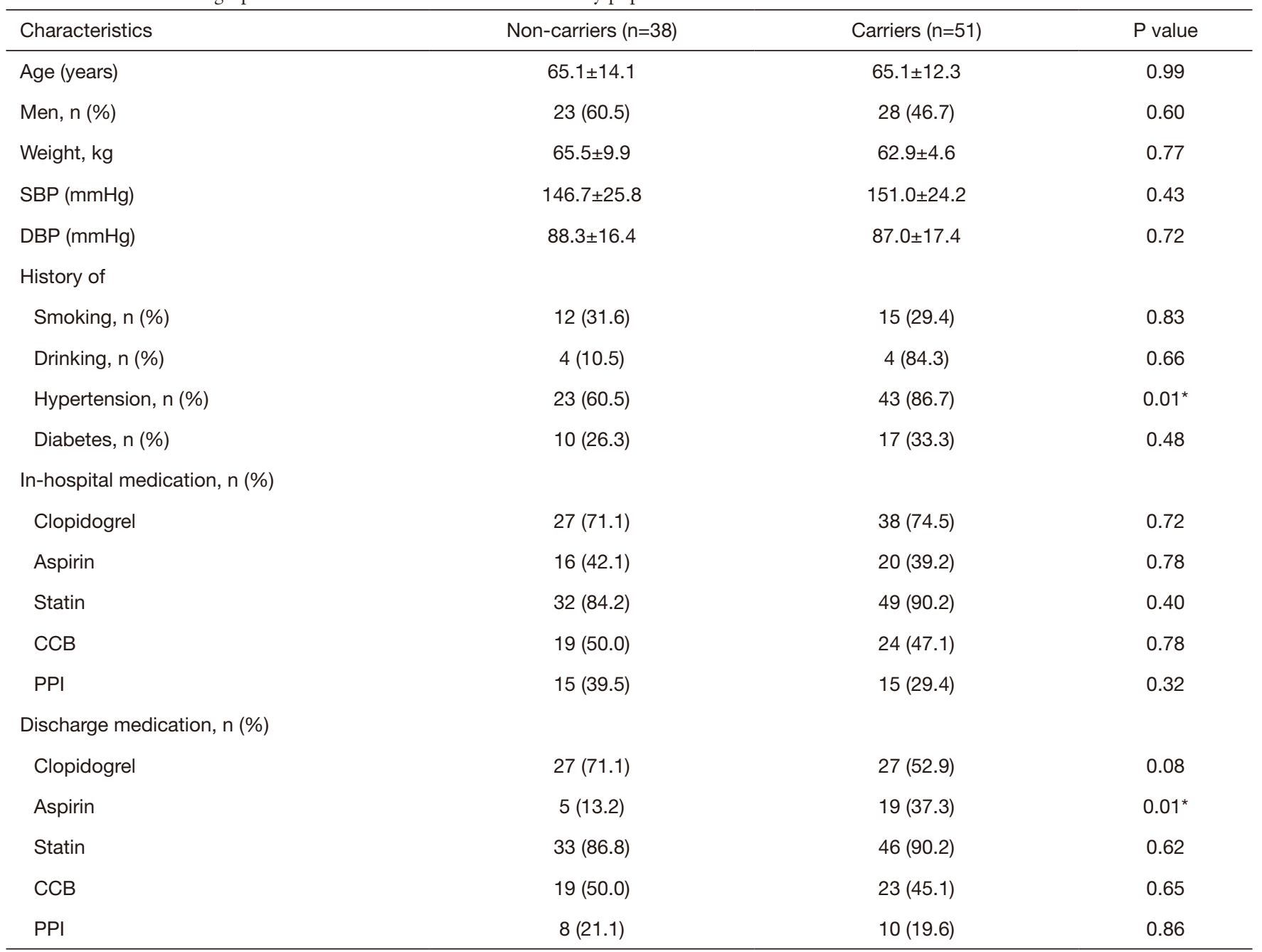

${ }^{*}, \mathrm{P}<0.05$ compared with non-carriers. SBP, systolic blood pressure; DBP, diastolic blood pressure; CCB, calcium channel blockers; PPI, proton pump inhibitor.

groups: non-carriers (including only CYP2C19*1/*1, n=38); and carriers of at least 1 CYP2C19 LOF allele (including CYP2C19*1/*2, $\left.{ }^{*} 1 / * 3,{ }^{*} 2 / * 2, * 2 / * 3, * 3 / * 3, n=51\right)$. Overall, the carriers of the LOF alleles had a more significant history of hypertension compared to the non-carriers $[n=43 / 51$ $(86.7 \%)$ versus $\mathrm{n}=23 / 38$ (60.5\%), $\mathrm{P}=0.01]$. In addition, the inclusion rate of aspirin in discharge medication was significantly higher for carriers than for non-carriers [ $\mathrm{n}=19 / 51$ (37.3\%) versus $\mathrm{n}=5 / 38(13.2 \%), \mathrm{P}=0.01$ ] (Table 2). None of the laboratory parameters showed a statistically significant difference in mean values between non-carriers and carriers (Table 3).

\section{The clinical follow-up}

All patients were monitored for 12 months. We found that the incidence of recurrent stroke in the groups carrying 1 or 2 LOF alleles was significantly higher than in non-carriers. The incidence of arteriosclerotic encephalopathy was also higher in the groups carrying 1 or 2 LOF alleles than in non-carriers. However, the occurrence of bleeding was not significantly different in any group (Table 4).

\section{Risk factors of recurrent stroke}

A multivariate analysis of sex, age, history of smoking, 
Table 3 Baseline laboratory parameters of the study population

\begin{tabular}{|c|c|c|c|}
\hline Laboratory values & Non-carriers $(n=38)$ & Carriers $(n=51)$ & $P$ value \\
\hline WBC $\left(10^{9} / \mathrm{L}\right)$ & $9.20 \pm 2.91$ & $10.05 \pm 3.92$ & 0.27 \\
\hline PLT $\left(10^{9} / L\right)$ & $227.76 \pm 69.29$ & $242.81 \pm 58.11$ & 0.28 \\
\hline FBG (mmol/L) & $6.98 \pm 3.00$ & $8.00 \pm 3.65$ & 0.19 \\
\hline Cholesterol (mmol/L) & $5.33 \pm 1.09$ & $5.56 \pm 1.56$ & 0.46 \\
\hline Triglyceride(mmol/L) & $1.97 \pm 1.58$ & $1.86 \pm 1.03$ & 0.68 \\
\hline TBIL $(\mu \mathrm{mol} / \mathrm{L})$ & $17.40 \pm 8.86$ & $15.67 \pm 7.5$ & 0.33 \\
\hline HDL-Cholesterol (mmol/L) & $1.19 \pm 0.25$ & $1.11 \pm 0.34$ & 0.23 \\
\hline $\mathrm{LP}(\mathrm{a})(\mathrm{mg} / \mathrm{L})$ & $195.75 \pm 71.60$ & $180.30 \pm 83.47$ & 0.83 \\
\hline $\mathrm{UA}(\mu \mathrm{mol} / \mathrm{L})$ & $389.05 \pm 101.77$ & $370.00 \pm 115.37$ & 0.43 \\
\hline $\mathrm{Cr}(\mu \mathrm{mol} / \mathrm{L})$ & $114.22 \pm 33.67$ & $108.60 \pm 26.62$ & 0.40 \\
\hline CysC (mg/L) & $1.07 \pm 0.24$ & $1.07 \pm 0.27$ & 0.96 \\
\hline
\end{tabular}

PT, prothrombin time; WBC, white blood cell; PLT, platelet; FBG, fasting blood-glucose; HbA1c, hemoglobin Alc; TBIL, total bilirubin; HCY, homocysteine; $\mathrm{LP}(\mathrm{a})$, lipoprotein(a); UA, uric acid; $\mathrm{Cr}$, creatinine; CysC, cystatin $\mathrm{C}$.

Table 4 Results of the clinical follow-up

\begin{tabular}{|c|c|c|c|c|}
\hline Variable & $\begin{array}{c}\text { Non-carriers } \\
\quad(n=38)\end{array}$ & \multicolumn{2}{|c|}{ Carriers $(n=51)$} & $P$ value \\
\hline Arteriosclerotic encephalopathy, n (\%) & $0(0)$ & $0(0)$ & $2(13.3)$ & $0.006^{\star \star}$ \\
\hline Recurrent stroke, n (\%) & $2(5.3)$ & $10(27.8)$ & $3(23.1)$ & $0.031^{*}$ \\
\hline Bleeding, n (\%) & $0(0)$ & $0(0)$ & $1(6.7)$ & 0.084 \\
\hline
\end{tabular}

**, $\mathrm{P}<0.01$ compared with non-carriers; ${ }^{*}, \mathrm{P}<0.05$ compared with non-carriers.

history of drinking, hypertension, diabetes, and CYP2C19 LOF alleles as risk factors for recurrent stroke (Table 5) revealed that while CYP2C19 LOF alleles were closely associated with an increased risk of recurrent stroke [odds ratio (OR): 0.072, 95\%; confidence interval (CI): 0.008$0.617 ; \mathrm{P}=0.016]$, the other factors were not.

\section{Discussion}

The CYP2C19 gene variant alleles play a crucial role in the metabolism and bioactivation of clopidogrel and its treatment outcomes in individuals. CYP2C19 LOF alleles impair the formation of active metabolites, resulting in reduced platelet inhibition (15). The most definitive studies demonstrating the relationship between the CYP2C19 genotype and the clopidogrel response have focused primarily on ACS patients, especially those undergoing a PCI $(14,16)$. However, several studies have also reported that CYP2C19 gene status can influence the risk of IS or other adverse clinical events following a stroke (17-19).

This study first evaluated the relationship between CYP2C19 polymorphisms and clopidogrel efficacy in patients with an IS in the Chaoshan region of China. Our results revealed the frequency of CYP2C19 EMs to be 
Table 5 Multivariate logistic regression analysis of risk predictors for recurrent stroke

\begin{tabular}{lccc}
\hline Covariate & OR & $95 \% \mathrm{Cl}$ & 0.828 \\
\hline Sex & 0.860 & $0.219-3.378$ & 0.666 \\
Age & 1.011 & $0.961-1.065$ & 0.153 \\
History of smoking & 3.179 & $0.651-15.510$ & 0.166 \\
History of drinking & 7.915 & $0.424-147.681$ & 0.998 \\
Hypertension & $1.164 \mathrm{E9}$ & $0.000-\infty$ & 0.934 \\
Diabetes & 1.062 & $0.257-4.387$ & $0.016^{*}$ \\
CYP2C19 LOF alleles & 0.072 & $0.008-0.617$ \\
\hline
\end{tabular}

${ }^{*} \mathrm{P}<0.05$.

45.08\%, IMs to be $37.71 \%$, and PMs to be $17.11 \%$. Clinical Pharmacogenetics Implementation Consortium (CPIC) guidelines have shown the frequencies of CYP2C19 EMs, IMs, PMs to be 35-50\%, 18-45\%, 2-15\%, respectively (15). Our study's EM and IM frequencies correspond to the CPIC guidelines. However, the frequency of PMs was higher in our study than the CPIC guidelines reported. This difference may be related to sample size and regional variations.

The CPIC guidelines indicate that the frequencies of CYP2C19*2 and CYP2C19*3 alleles are 29-35\% and 2-9\% in Asian populations. In our study, the allelic frequency of CYP2C19*2 was $31.97 \%$, and that of CYP2C19*3 was $4.10 \%$. Furthermore, a study of the Chinese population reported CYP2C19*2 and CYP2C19*3 allele frequencies of $31.80 \%$ and $5.06 \%$, respectively (20), which is close to our results.

Epidemiological studies have demonstrated that high blood pressure (BP) is the most important risk factor for stroke (2). Stroke risk is close to linear, beginning at relatively low BP levels $(21,22)$. High BP increases both the risk of stroke and death following a stroke (23). Numerous trials have shown a reduction in stroke risk with hypertension treatment and lower rates of recurrent stroke with lower BPs (24-26). In our study, carriers of LOF alleles have a more significant history of hypertension than non-carriers, which indicates that active antihypertensive treatment is important for carriers of CYP2C19 LOF alleles suffering an IS and in the prevention of recurrent strokes.

This study found that the rate of aspirin inclusion in the discharge medication of CYP2C19 LOF alleles carriers was higher than for non-carriers. Aspirin reduces stroke mortality by preventing thrombotic IS (2). A meta-analysis of trials found that aspirin could reduce the risk of a stroke incident when used for secondary prevention of IS (27). However, the results of other trials reported no benefits of prescribing aspirin for the primary prevention of stroke in the general population $(6,28,29)$. To date, many studies have focused on aspirin in the primary or secondary prevention of IS, but not on the benefits of aspirin for CYP2C19 LOF alleles carriers. We believe aspirin can be used instead of clopidogrel for secondary prevention of stroke, especially for carriers of 1 or 2 CYP2C19 LOF alleles.

Previous studies have demonstrated that carriers of 1 or 2 CYP2C19 LOF alleles present significantly lower levels of the active metabolite of clopidogrel and a higher rate of recurrent cerebrovascular events $(30,31)$. One study also reported that carrying CYP2C19 LOF alleles has an important impact on the response to clopidogrel and prognosis in patients with IS (32). Another study in China indicated that CYP2C19 LOF alleles could increase the risk of recurrent IS events (19). After multivariate logistic regression analysis, the present study found that CYP2C19 $\mathrm{LOF}$ alleles are related to and could be an independent risk factor for recurrent stroke.

Therefore, it is worth emphasizing that CYP2C19 genotyping might be more reliable and helpful to guide individualized therapy, thus optimizing therapeutic plans which are vitally important for improving stroke prognosis and possible preventing of stroke recurrence in patients. Clinical treatment alternatives to prevent recurrent adverse cardiac outcomes include increasing the clopidogrel dose, adding other agents, or switching to another medication, such as prasugrel or ticagrelor $(8,30)$. So, patients with CYP2C19 LOF alleles also can use these treatment alternatives, such as acetylsalicylic acid-dipyridamole, which was shown to prevent recurrent strokes. Further, the warning on clopidogrel suggests that clinicians consider 
other anti-platelet treatments in patients known to be CYP2C19 PMs (8).

There are several important limitations in our study, which may lead to possible bias. These include small sample size and a short follow-up period. Moreover, although we focused on the relationship between CYP2C19 polymorphisms and IS prevention, we did not investigate whether CYP2C19 polymorphisms, especially CYP2C19 LOF alleles, covalently contributed to clopidogrel resistance. Therefore, future studies should use a larger sample size and consider modeling to clarify the relationship between CYP2C19 polymorphisms and clopidogrel resistance.

In conclusion, the findings of this retrospective observational study demonstrate that patients who have had an IS and carry CYP2C19 LOF alleles (*2 and *3) may be at greater risk of recurrent strokes. The polymorphisms of CYP2C19 may be predictors of a poor functional outcome in patients with recurrent strokes. Like other antiplatelet medications, aspirin can be prescribed instead of clopidogrel for secondary stroke prevention in carriers of CYP2C19 LOF alleles.

\section{Acknowledgments}

Funding: This study was supported by the Science and Technology Planning Project of Shantou, China (No. 190603085267898 and No. 180319234014210 ), the Medical Scientific Research Foundation of Guangdong Province, China (No. B2021142) and the Beijing Bethune Charitable Foundation, China (No. AE012DS).

\section{Footnote}

Reporting Checklist: The authors have completed the STROBE reporting checklist. Available at https://dx.doi. org/10.21037/apm-21-2905

Data Sharing Statement: Available at https://dx.doi. org/10.21037/apm-21-2905

Conflicts of Interest: All authors have completed the ICMJE uniform disclosure form (available at https://dx.doi. org/10.21037/apm-21-2905). The authors have no conflicts of interest to declare.

Ethical Statement: The authors are accountable for all aspects of the work in ensuring that questions related to the accuracy or integrity of any part of the work are appropriately investigated and resolved. The study was approved by ethics board of the First Affiliated Hospital of Shantou University Medical College, China (No. 2019043). The study was conducted in accordance with the Declaration of Helsinki (as revised in 2013) and was consistent with relevant guidelines and regulations for clinical studies. Informed consent was obtained from each patient before enrollment.

Open Access Statement: This is an Open Access article distributed in accordance with the Creative Commons Attribution-NonCommercial-NoDerivs 4.0 International License (CC BY-NC-ND 4.0), which permits the noncommercial replication and distribution of the article with the strict proviso that no changes or edits are made and the original work is properly cited (including links to both the formal publication through the relevant DOI and the license). See: https://creativecommons.org/licenses/by-nc-nd/4.0/.

\section{References}

1. Chen F, Qi Z, Luo Y, et al. Non-pharmaceutical therapies for stroke: mechanisms and clinical implications. Prog Neurobiol 2014;115:246-69.

2. Lackland DT, Roccella EJ, Deutsch AF, et al. Factors influencing the decline in stroke mortality: a statement from the American Heart Association/American Stroke Association. Stroke 2014;45:315-53.

3. Wang W, Jiang B, Sun H, et al. Prevalence, Incidence, and Mortality of Stroke in China: Results from a Nationwide Population-Based Survey of 480687 Adults. Circulation 2017;135:759-71.

4. Wu X, Liang Y, Zheng B, et al. Care stress in caregivers of disabled stroke patients: a cross-sectional survey. Ann Palliat Med 2020;9:2211-20.

5. Meschia JF, Bushnell C, Boden-Albala B, et al. Guidelines for the primary prevention of stroke: a statement for healthcare professionals from the American Heart Association/American Stroke Association. Stroke 2014;45:3754-832.

6. Wang YJ, Wang CX, Miao ZR. Guidelines for secondary prevention of ischemic stroke and transient ischemic attack in China 2014. Chinese Journal of Neurology 2015;48:258-73.

7. Simmons BB, Yeo A, Fung K; American Heart Association; 
American Stroke Association. Current guidelines on antiplatelet agents for secondary prevention of noncardiogenic stroke: an evidence-based review. Postgrad Med 2010;122:49-53.

8. Perry CG, Shuldiner AR. Pharmacogenomics of antiplatelet therapy: how much evidence is enough for clinical implementation? J Hum Genet 2013;58:339-45.

9. McDonough CW, McClure LA, Mitchell BD, et al. CYP2C19 metabolizer status and clopidogrel efficacy in the Secondary Prevention of Small Subcortical Strokes (SPS3) study. J Am Heart Assoc 2015;4:e001652.

10. Sangkuhl K, Klein TE, Altman RB. Clopidogrel pathway. Pharmacogenet Genomics 2010;20:463-5.

11. Amin AM, Sheau Chin L, Azri Mohamed Noor D, et al. The Personalization of Clopidogrel Antiplatelet Therapy: The Role of Integrative Pharmacogenetics and Pharmacometabolomics. Cardiol Res Pract 2017;2017:8062796.

12. Jiang XL, Samant S, Lesko LJ, et al. Clinical pharmacokinetics and pharmacodynamics of clopidogrel. Clin Pharmacokinet 2015;54:147-66.

13. Brown SA, Pereira N. Pharmacogenomic Impact of CYP2C19 Variation on Clopidogrel Therapy in Precision Cardiovascular Medicine. J Pers Med 2018;8:8.

14. Mega JL, Simon T, Collet JP, et al. Reduced-function CYP2C19 genotype and risk of adverse clinical outcomes among patients treated with clopidogrel predominantly for PCI: a meta-analysis. JAMA 2010;304:1821-30.

15. Scott SA, Sangkuhl K, Stein CM, et al. Clinical Pharmacogenetics Implementation Consortium Guidelines for CYP2C19 Genotype and Clopidogrel Therapy: 2013 Update. Clin Pharmacol Ther 2013;94:317-23.

16. Holmes MV, Perel P, Shah T, et al. CYP2C19 genotype, clopidogrel metabolism, platelet function, and cardiovascular events: a systematic review and metaanalysis. JAMA 2011;306:2704-14.

17. Yi X, Lin J, Wang Y, et al. Association of Cytochrome P450 Genetic Variants with Clopidogrel Resistance and Outcomes in Acute Ischemic Stroke. J Atheroscler Thromb 2016;23:1188-200.

18. Pan Y, Chen W, Xu Y, et al. Genetic Polymorphisms and Clopidogrel Efficacy for Acute Ischemic Stroke or Transient Ischemic Attack: A Systematic Review and MetaAnalysis. Circulation 2017;135:21-33.

19. Wang Y, Cai H, Zhou G, et al. Effect of CYP2C19*2 and *3 on clinical outcome in ischemic stroke patients treated with clopidogrel. J Neurol Sci 2016;369:216-9.

20. Zhu WY, Zhao T, Xiong XY, et al. Association of CYP2C19 Polymorphisms with the Clinical Efficacy of Clopidogrel Therapy in Patients Undergoing Carotid Artery Stenting in Asia. Sci Rep 2016;6:25478.

21. Lewington S, Clarke R, Qizilbash N, et al. Age-specific relevance of usual blood pressure to vascular mortality: a meta-analysis of individual data for one million adults in 61 prospective studies. Lancet 2002;360:1903-13.

22. James PA, Oparil S, Carter BL, et al. 2014 evidence-based guideline for the management of high blood pressure in adults: report from the panel members appointed to the Eighth Joint National Committee (JNC 8). JAMA 2014;311:507-20.

23. Kuller LH. Epidemiology and prevention of stroke, now and in the future. Epidemiol Rev 2000;22:14-7.

24. Mochizuki S, Dahlöf B, Shimizu M, et al. Valsartan in a Japanese population with hypertension and other cardiovascular disease (Jikei Heart Study): a randomised, open-label, blinded endpoint morbidity-mortality study. Lancet 2007;369:1431-9.

25. Schrader J, Lüders S, Kulschewski A, et al. Morbidity and Mortality After Stroke, Eprosartan Compared with Nitrendipine for Secondary Prevention: principal results of a prospective randomized controlled study (MOSES). Stroke 2005;36:1218-26.

26. Weber MA, Bakris GL, Jamerson K, et al. Cardiovascular events during differing hypertension therapies in patients with diabetes. J Am Coll Cardiol 2010;56:77-85.

27. Antithrombotic Trialists' Collaboration. Collaborative meta-analysis of randomised trials of antiplatelet therapy for prevention of death, myocardial infarction, and stroke in high risk patients. BMJ 2002;324:71-86.

28. Raju N, Sobieraj-Teague M, Hirsh J, et al. Effect of aspirin on mortality in the primary prevention of cardiovascular disease. Am J Med 2011;124:621-9.

29. Antithrombotic Trialists' (ATT) Collaboration; Baigent C, Blackwell L, et al. Aspirin in the primary and secondary prevention of vascular disease: collaborative meta-analysis of individual participant data from randomised trials. Lancet 2009;373:1849-60.

30. Jia DM, Chen ZB, Zhang MJ, et al. CYP2C19 polymorphisms and antiplatelet effects of clopidogrel in acute ischemic stroke in China. Stroke 2013;44:1717-9.

31. Spokoyny I, Barazangi N, Jaramillo V, et al. Reduced clopidogrel metabolism in a multiethnic population: prevalence and rates of recurrent cerebrovascular events. J 
Stroke Cerebrovasc Dis 2014;23:694-8.

32. Qiu LN, Sun Y, Wang L, et al. Influence of CYP2C19 polymorphisms on platelet reactivity and clinical outcomes in ischemic stroke patients treated with clopidogrel. Eur J Pharmacol 2015;747:29-35.

Cite this article as: Lin J, Mo Y, Cai D, Mao D, Fu H, Wei D. CYP2C19 polymorphisms and clopidogrel efficacy in the secondary prevention of ischemic stroke: a retrospective observational study. Ann Palliat Med 2021;10(12):12171-12180. doi: 10.21037/apm-21-2905 